\title{
Numerical Simulation of Sound Radiated from a Turbulent Vortex Ring
}

\author{
Hongyu Ran* and Tim Colonius ${ }^{\dagger}$ \\ California Institute of Technology, Pasadena, California 91125
}

\begin{abstract}
The acoustic field radiated by a turbulent vortex ring is studied. Direct Numerical Simulations (DNS) of the fully compressible, three-dimensional Navier-Stokes equations are used to generate an axisymmetric vortex ring to which $3 D$ stochastic disturbances are added. The disturbances cause instability and turbulent transition of the vortex ring. Detailed information about temporal evolution of sound pressure level, spectrum and directivity associated with each mode is investigated. The peak frequency agrees well with experiments, and the directivity of each azimuthal mode agrees well with predictions of vortex sound theory. Based on the self-similar decay of the turbulent near field, the selfsimilar decay of the sound field is investigated. We also explore the connections with jet noise by modeling the jet as a de-correlated train of vortex rings.
\end{abstract}

\section{Introduction}

It has been suggested that jet noise can be decomposed into two elements: the noise radiated by largescale structures, and that radiated by fine-scale turbulence. ${ }^{1}$ Statistical models for the fine-scale component need to be combined with models for the deterministic noise produced by coherent structures. Thus the goal of understanding sound generation by large scales is a motivation of the present work. A turbulent vortex ring has interesting connections with turbulent jets, and is also comprised of both components, i.e. large-scale vorticity structure (eigensolutions) and fine-scale turbulence. In the core region, the turbulence is suppressed, and many families of unstable modes can be excited. These different modes produce a peak in the noise spectra.

A vortex ring can be generated in the laboratory by the motion of a piston pushing a column of fluid through an orifice or nozzle. Glezer ${ }^{2}$ categorizes the generating conditions for vortex rings and classifies the conditions under which a given vortex generator produces either an initially laminar or turbulent ring. A transition map is constructed, which is based on two parameters: $R e=\Gamma / \nu$, and stroke length $L / D$. For a moderate stroke number $L / D=1$, the critical Reynolds number is about $10^{4}$. With moderate Reynolds number, a laminar vortex ring will be generated initially, but the vortex ring is unstable to azimuthal disturbance, and will break down into a turbulent vortex ring.

The transition from laminar to turbulent flow in a vortex ring begins with azimuthal instabilities that have been studied experimentally ${ }^{3,4}$ and theoretically. ${ }^{5,6}$ The linear instability and the subsequent nonlinear amplification stage can be investigated with numerical simulations. ${ }^{7}$ The onset of turbulence in the vortex ring was studied with vortex methods. ${ }^{8}$

Among experimental studies of the turbulent vortex ring, Glezer and Coles ${ }^{9}$ investigated the self similar properties of the ensemble averaged flow. When the flow is turbulent, there will be dispersion in the

*Research Assistant, Division of Engineering and Applied Science, California Institute of 'Technology, Pasaderna, California 91125.

†Associate Professor, Division of Engineering and Applied Science, California Institute of Techrology, Pasaderia, California 91125, Memeber, AIAA. 
trajectories of individual rings, and ensemble averages must be carefully implemented. Profiles of axial mean velocity, radial mean velocity, and Reynolds stress were measured. Weigand and Gharib ${ }^{10}$ and Auerbach ${ }^{11}$ observed periodic shedding of vorticity from the vortex ring to the wake, which results in the reduction of Reynolds number. The first experimental investigation of sound generated by a turbulent vortex ring was reported by Zaitsev, Kopiev, Munin and Potokin. ${ }^{12}$ They found that the sound field is localized in a narrow frequency band, and observed a drift of the peak frequency in the spectrum toward lower frequency as the vortex ring evolves. In a more recent experiment, Zaitsev, Kopiev and Kotova ${ }^{13}$ used a circular array of microphones in order to extract quadrupole components from the total noise. Average contributions of different azimuthal components were studied, and their directivity was compared with prediction from vortex sound theory. ${ }^{14}$ However, since the microphone array was placed in one circular plane, instantaneous polar distribution of acoustic signal could not be measured, and had to be obtained from the analysis of the time evolution of different harmonics. The nonstationary character of the mean flow complicated the analysis.

To model the sound generation problem, Shariff, Leonard and Ferziger ${ }^{15}$ used an axisymmetric ring with an elliptical core to study the acoustic signal. The non-circular core structure will cause oscillation in both aspect ratio and orientation angle. Using vortex sound theory, the acoustic signal is found to be sinusoidal at twice the frequency of the core rotation. The problem was also studied using contour dynamics, ${ }^{16,17}$ and it is found that the two basic vortex sound generation mechanisms are vortex core deformation and the vorticity centroid dynamics, and are independent of the specific vortex system.

Kopiev and Chernyshev ${ }^{18}$ used a thin-core vortex ring model to find eigen-oscillations of the core, and investigated mechanisms that produce sound using vortex sound theory. The most efficient radiation arose from the axisymmetric and first two azimuthal modes. The theory also explained several experimental observations about the sound spectrum.

In the present study, the acoustic field radiated by the turbulent vortex ring is solved simultaneously with the flow field. In the first part, we study the turbulent transition process, including the nonlinear amplification of the higher azimuthal modes, breaking of the azimuthal waves, and the instantaneous vortical structure of the turbulent vortex ring. Self-similarity of the turbulent flow is evaluated by averaging an ensemble of simulations. In the second part, we investigate the acoustic field generated by the vortex motion, with focus on frequency spectrum, sound pressure level, and directivity of the acoustic signal with different azimuthal numbers. The sound field of a train of de-correlated vortex rings is studied and compared with the sound field of turbulent jets with similar Mach number and Reynolds number.

\section{Computational Technique}

The 3-D unsteady compressible Navier-Stokes equations were solved in a cylindrical coordinate system using a sixth-order compact finite difference scheme ${ }^{19}$ for the axial and radial directions and Fourier spectral differentiation in the azimuthal direction. For time advancement, an explicit fourth-order Runge-Kutta method is used. At the boundary, a sponge zone ${ }^{20}$ is used to absorb the waves, and the polar coordinate centerline treatment proposed by Mohseni and Colonius ${ }^{21}$ is used. The equations were discretized with as many as $240 \times 120 \times 128$ mesh points in the axial $(x)$, radial $(r)$, and azimuthal $(\theta)$ directions, respectively. The computational domain is shown schematically in Figure 1. From the nominal center of the vortex ring, the domain extends to about $-12 R$ and $12 R$ in the axial direction and $12 R$ in the radial direction, where $R$ is the initial radius of the vortex ring. The grid spacing is $\frac{1}{33} R$ in the vortex ring region, and is stretched to a maximum $\frac{1}{2.5} R$ near the boundary. The time step is $0.012 R / a$ where $a$ is the sound speed. The code is parallelized to run on a 32 node Beowulf cluster.

The far field is extended by solving a wave equation in a spherical coordinate system $(|x|, \chi, \theta)$, where $|x|$ is the radial distance to the vortex ring, $\chi$ is the polar angle, and $\theta$ the azimuthal angle. The DNS solution on a sphere of radius $10 R$ is decomposed into spherical harmonics $Y_{l}^{m}(\chi, \theta)$, where $m$ and $l$ are azimuthal number and polar number, respectively. A one-dimension wave equation in the radial direction is solved for each spherical harmonic on a domain $10 R<|x|<70 R$.

A non-conservative body force is applied to the equations of motion for a period of time $T$ to generate the. 
vortex ring. Vorticity is deposited in a layer with radius $R$ that rolls up into a thicker ring with controlled parameters, e.g. ring radius, circulation, etc. The same method was used by Mohseni, Ran and Colonius ${ }^{22}$ to study vortex ring pinch-off. As shown in that paper, the flow properties are very similar to vortex rings generated with the cylinder/piston mechanism.

In what follows, we characterize the ring by its properties upon completion of the formation stage. There are four nondimensional parameters that control the formed ring: the ratio of core to ring radius, $\sigma / R$; the circulation of the formed vortex ring, $\Gamma T ; R^{2}$, which can be mapped to a specific stroke length to diameter ratio $L / D$ in the cylinder/piston mechanism; the circulation-based Reynolds number, $\Gamma / \nu$ (where $\nu$ is the constant kinematic viscosity), and a Mach number, for example $\Gamma / R a_{\infty}$. For convenience, we define a nominal translation Mach number $M$ that is based on the estimated average translation speed of the ring. With the chosen forcing parameters in the current studies, the corresponding stroke length is about $L / D=1.5$, and the nominal translational Mach number of the vortex ring is varied from 0.14 to 0.28 . Once formed, the laminar vortex ring has an initial Reynolds number $\Gamma / \nu=4500$, and a core thickness $\sigma / R=0.34$. Unless otherwise noted, quantities are made nondimensional with circulation $\Gamma$ and vortex ring radius $R$. For example, the nondimensional time is defined as $\hat{t}=t \Gamma / R^{2}$.

In the present numerical studies, we add a uniform flow with speed $M$ in the direction opposite to ring translation. In this way the vortex structure is (approximately) centered in the computation domain. Azimuthal instability is introduced by adding azimuthal perturbations to the spatial distribution of the forcing function. This is equivalent to imposing a random radial displacement on the toroidal radius of the ring $r^{\prime}(\theta)=r_{0}[1+\varepsilon(\theta)]$ (see Figure 1). It should be noted that the disturbance field is divergence free in order to minimize the sound generated by the forcing. When $\varepsilon$ is too large, nonlinear effect appears right after vortex ring formation. We found that when $\varepsilon$ is less than 0.02 , the growth rate of the linear instability is independent of the perturbation level. In the simulations presented here, we use $\varepsilon=0.01$. Both the flow field and the sound field are ensemble averaged with as many as 12 ensembles to obtain mean flow quantities and sound spectrum.

Resolution studies with reduced grid size of $160 \times 80 \times 64$ shows that at this Reynolds number, the transition and turbulent stages of the vortex ring are well resolved on the $240 \times 120 \times 128$ mesh.

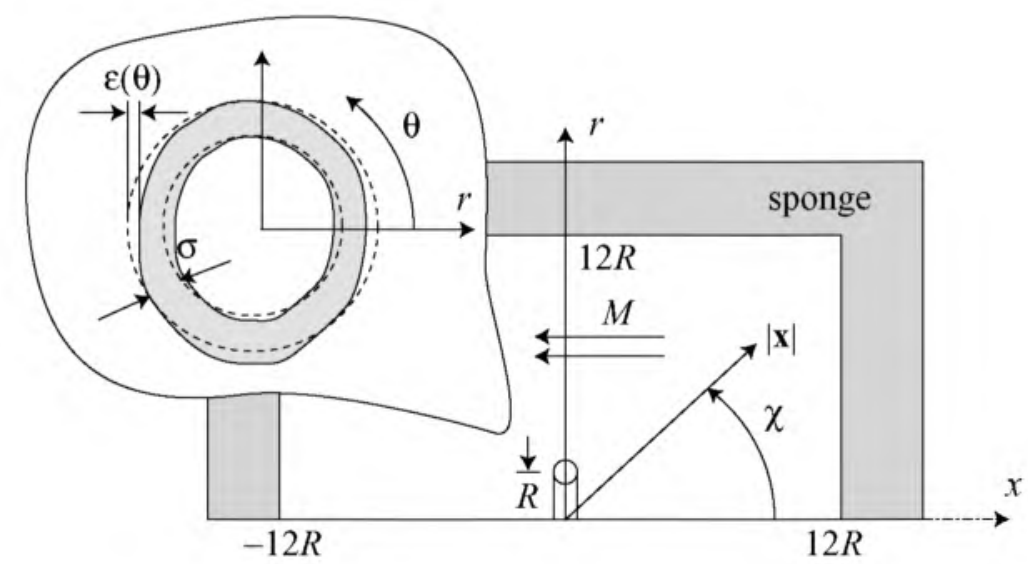

Figure 1. Schematic diagram of computational domain for vortex ring. 


\section{Results and Discussion}

The evolution of the vortex ring can be roughly separated into 4 stages, which are depicted in Figure 2 (a) for a ring with $M=0.28$. During stage I, the vortex ring is formed by application of the non-conservative body force, and the circulation grows linearly. The forcing also produces a spherically spreading dipolar acoustic wave. By applying the force slowly on the acoustic timescale, the amplitude of this acoustic transient is minimized. During stage II, three-dimensional instabilities occur while the overall circulation decays slightly due to (laminar) viscous diffusion across the centerline. Depending on the rate at which the body force is applied, there is overlap between stages I and II with formation and instabilities occurring simultaneously. Toward the end of stage II, nonlinear interactions of the unstable modes cause transition to turbulent flow. During stage III, the flow is turbulent and circulation decays at higher rate due both to viscous diffusion and cancellation of opposite-signed vorticity across the ring axis (brought about by turbulent mixing and the shedding of vorticity from the ring). The decay approximately follows a $t^{1 / 2}$ law predicted by Glezer and Coles. ${ }^{9}$ As the circulation of the ring decays, its translational velocity becomes smaller than the nominal translation velocity and the ring moves upstream relative to the computational domain. During the final stage IV, part of the vortex ring exits the computational domain and the circulation decreases rapidly.

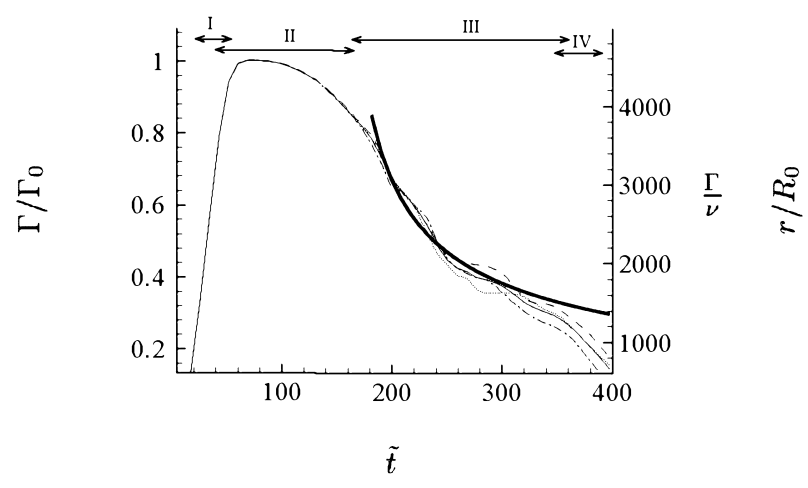

(a)

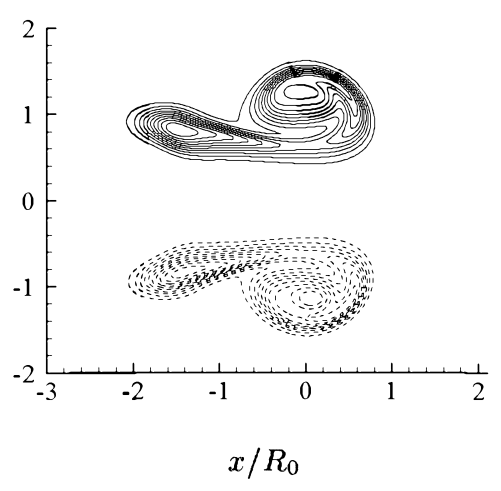

(b)

Figure 2. (a) Decaying of total circulation and Reynolds number of 4 vortex ring realizations. The thicker line is $\sim \tilde{t}^{-1 / 2}$. Stage I, II, III and IV are described in this section; (b) side view of azimuthal vorticity at $\theta=0$ and $\theta=\pi$ just after formation, with 20 evenly spaced contours between -2.4 and $2.4, M=0.28, \tilde{t}=43.2$.

\section{A. Three-dimensional Vortex Ring Instabilities}

Linear oscillation modes of the ring can be characterized by azimuthal wave number $n$, radial mode number $j$ and rotation rate $\Omega$. In addition, the vortex core is strained caused by the geometry of the ring. If the rotation rate $\Omega$ is smaller than the strain rate $\epsilon$, the mode become unstable. When the flow is viscous, higher radial modes will usually be damped and the unstable modes with the least radial structure will be amplified.

To study the instability process and compare with theory, a vortex ring with $M=0.14$ is simulated; the forcing time is made shorter in order to separate the ring formation (stage I) and the instabilities (stage II). The instability can be visualized with contours of azimuthal vorticity shown in Figure 3. At earlier times $\tilde{t}=3.7$ and 15.0 , only the boundary of the vortex core is distorted, the radial profile of vorticity has only one peak, and no azimuthal mode is dominant. However, after time $\tilde{t}=30.0$, the radial profile of vorticity 
develops two peaks, illustrating the second radial mode. At one section, the contours in the inner region of the core are bent toward the ring axis, whereas contours at the outer region are bent outward. The azimuthal mode $n=7$ begins to dominant. This mode is non-rotating, but its amplitude increase in time. At time $\tilde{t}=$ 71.2 , the distortion of the iso-vorticity contours become comparable with the core radius, and the instability reaches the nonlinear phase. The peak vorticity level also increases due to the concentration of vorticity.

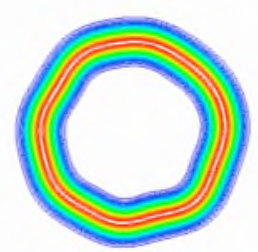

(a)

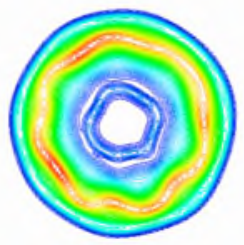

(d)

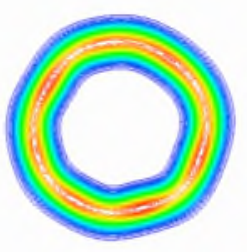

(b)

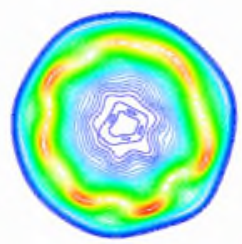

(e)

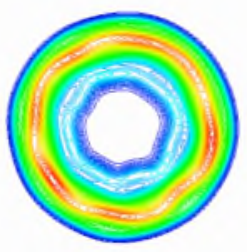

(c)

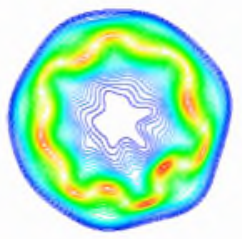

(f)

Figure 3. Contours of azimuthal vorticity on a plane across the ring axis. $\vec{t}=$ (a) 3.7, (b) 15.0, (c) 30.0, (d) 45.0 , (e) 60.0 , (f) 71.2 , with contour levels in a): $\max .=0.022$, $\min .=0.001$, increment $=0.001$; in (b) to (f), $\max .=0.7, \min .=0.02$, increment $=0.02$.

According to the heuristic linear instability model of Widnall and Tsai, ${ }^{5}$ a particular azimuthal number is unstable only for a certain radial mode. For example, $n=5$ should be unstable to a second radial mode $(j=2), n=9$ and 10 should be unstable to the third radial mode $(j=3)$. Figure 4 shows an end view of the contours of azimuthal vorticity at $\tilde{t}=71.2$, showing the radial structure of each azimuthal mode. The group $n=4$ to 8 has structure with $j=2$, and $n=9$ to 10 shows $j=3$. The contour levels show that modes $n=6$ and 7 are dominant. According to the inviscid theory, the most amplified azimuthal wave number of a non-rotating second-radial mode is given by $n=2.51 R / \sigma$; for $\sigma / R=0.34, n=7.4$, which agrees with the numerical result.

To demonstrate the temporal evolution of the instability waves, the kinetic energy associated with different azimuthal modes are plotted in figure 5. Up to time $\vec{t}=75$, the evolution goes through three phases: transient, linear, and early nonlinear. All the modes start with the same disturbance level, and oscillate during the early transient stage. However, after $\tilde{t}=30$, linearly growing eigenmodes $\mathrm{n}=6,7,8$ begin to dominant, whereas other modes start to decay. The most amplified mode during the linear stage is $n=7$. These unstable modes extract energy from the axisymmetric mean flow, and feed into other modes during the later nonlinear stage.

\section{B. Turbulent Transition}

During the nonlinear stage, several phenomena occur: harmonics of the most linearly amplified waves (e.g. $n=12$ to 16,24 to 32 , etc.) grow with higher growth rates; lower azimuthal modes arise from the nonlinear 


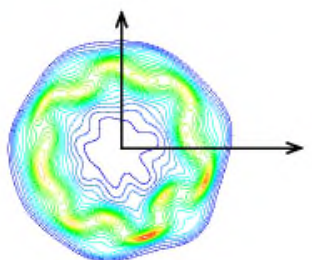

total

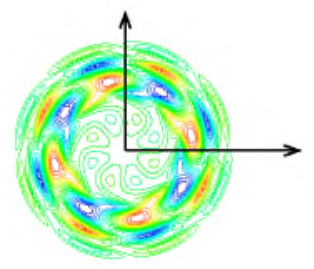

$\mathrm{n}=4$

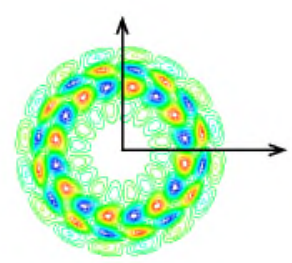

$\mathrm{n}=8$

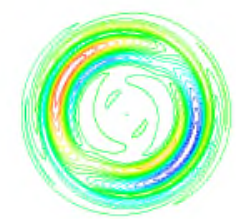

$\mathrm{n}=1$

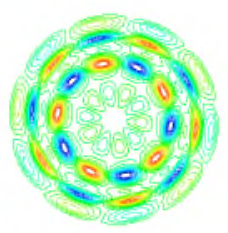

$\mathrm{n}=5$

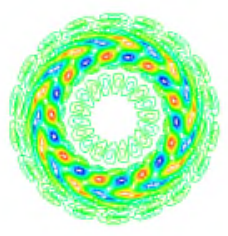

$\mathrm{n}=9$

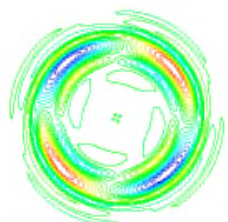

$\mathrm{n}=2$

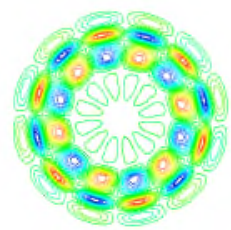

$\mathrm{n}=6$

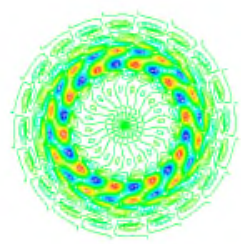

$\mathrm{n}=10$

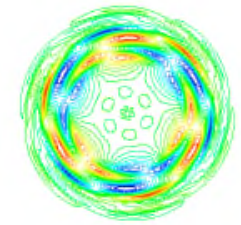

$\mathrm{n}=3$

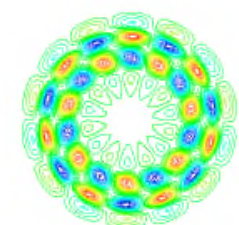

$n=7$

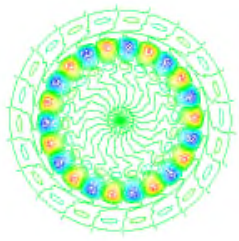

$\mathrm{n}=11$

Figure 4. Mode shape plots of $\omega_{\theta}$ at $\hat{t}=71.2$, with 20 contours between $\omega_{\max }$ and $-\omega_{\max }$ except for total vorticity. Total: $\min .=0.04, \max .=0.84, n=1$ to $11: \omega_{\max }=0.068,0.065,0.028,0.048,0.071,0.13,0.11,0.071$, $0.015,0.011,0.037$.

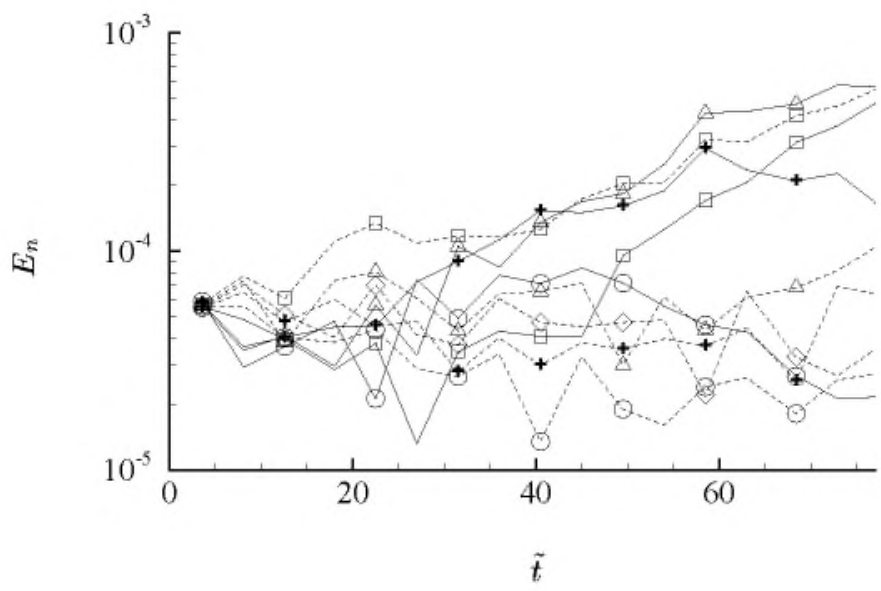

Figure 5. Evolution of kinetic energy. (----), $n=1$ to $5:(\square), 1 ;(\triangle), 2 ;(\diamond), 3 ;(+), 4 ;(\circ), 5 ;(\square), n=6$ to $9:(\square), 6 ;(\triangle), 7 ;(+), 8 ;(\circ), 9$. 
interaction of adjacent unstable modes; and the three dimensional disturbance of vorticity leads to the growth of an azimuthal velocity and stream-wise vorticity.

The process is demonstrated in Figure 6. Six waves appears around the vortex ring during the linear instability stage. At $\tilde{t}=86.4$, a harmonic with $n=12$ with higher radial mode causes the bulging shape in the isosurface. Eventually, its vorticity is unevenly distributed around the ring, leading to breakdown and transition. In addition, regions with lower vorticity around the periphery of the vortex ring form hairpin vortices and start to detach from the vortex ring, as depicted in Figure 6 (e) (f).
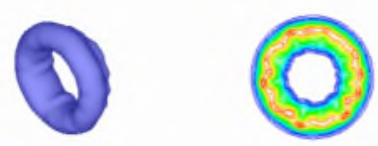

(a)

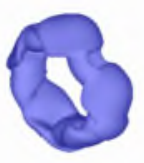

$(d)$
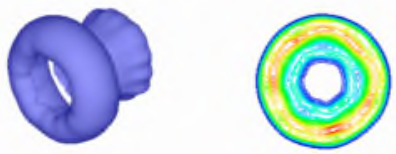

(b)

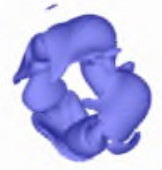

$(e)$

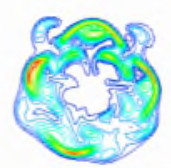

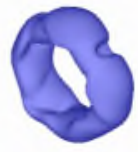

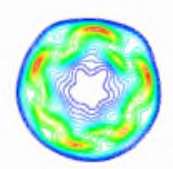

(c)
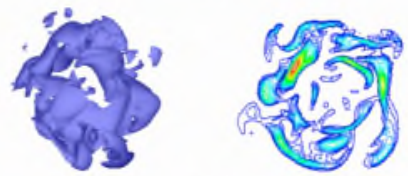

$(f)$

Figure 6. Left, isosurface of vorticity level 0.5 at $\tilde{t}=25.9,43.2,86.4,103.6,129.6,172.8$. Right, end view of vorticity with 20 evenly spaced contours between 0 and the maximum vorticity. $\omega_{\max }=$, a) 1.6, b) 1.2 , c) 1.3 , b) 1.6, e) 1.6, f) 2.2 .

Ensemble averaged one-dimensional energy spectra in azimuthal direction are shown in Figure 7 (a). In the initial stage, only wave numbers from 1 to 32 are excited, and higher wave numbers start with zero energy. The energy distribution reaches a quasi-equilibrium state after time $\tilde{t}=110$, representing the completion of turbulent transition, and the flow is well mixed by the turbulence. The overall energy spectrum decays in time due to turbulent dissipation. For more detailed study of vortex ring transition please refer to Ran. ${ }^{23}$

\section{Turbulent Statistics and Self-similarity}

In this section, 12 realizations for the case $M=0.28$ are ensemble averaged to obtain the average flow properties and turbulence statistics. Figure 7 (b) plots the translational velocity of the centroid of the vortex rings. The decay in velocity follows a stair-like fashion, similar to that reported by Weigand and Gharib. ${ }^{10}$ It is caused by the periodic shedding of vorticity from the vortex ring into the wake.

In a fully turbulent vortex ring, the evolution of statistical quantities will be independent of the kinematic viscosity and depend only on linear impulse $I / \rho$, where $I$ is computed from the time integral of the forcing function. Then from dimensional analysis we can define a new velocity scale, $u_{s}$, and nondimensional coordinates $\xi$ and $\eta$ with, ${ }^{9}$

$$
u_{s}=(I / \rho)^{\frac{1}{4}}\left(\tilde{t}-\tilde{t}_{0}\right)^{-\frac{3}{4}}, \quad \xi=\left(x-x_{0}\right)\left(\frac{\rho}{I\left(\tilde{t}-\tilde{t}_{0}\right)}\right)^{\frac{1}{4}}, \quad \eta=r\left(\frac{\rho}{I\left(\tilde{t}-\tilde{t}_{0}\right)}\right)^{\frac{1}{4}},
$$

which gives a power law,

$$
u_{p}^{-\frac{1}{3}} \sim\left(\tilde{t}-\tilde{t}_{0}\right)^{\frac{1}{4}} \sim\left(x-x_{0}\right) .
$$

where $x_{0}$ and $\tilde{t}_{0}$ are virtual origins of the turbulent vortex rings, $u_{p}$ is the peak axial velocity along the centerline. 


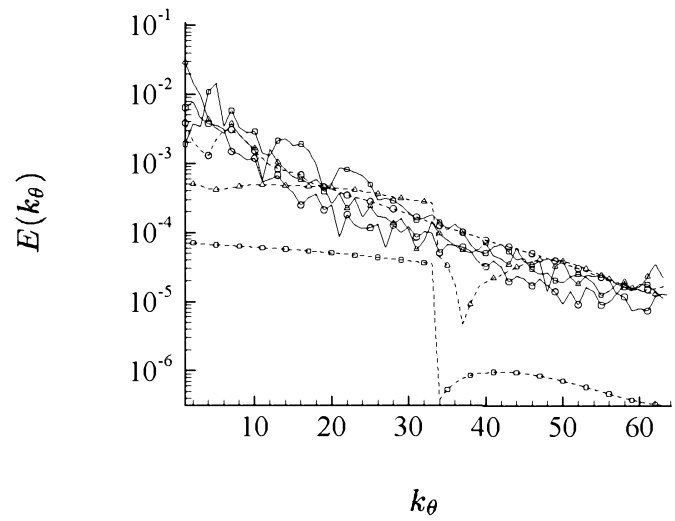

(a)

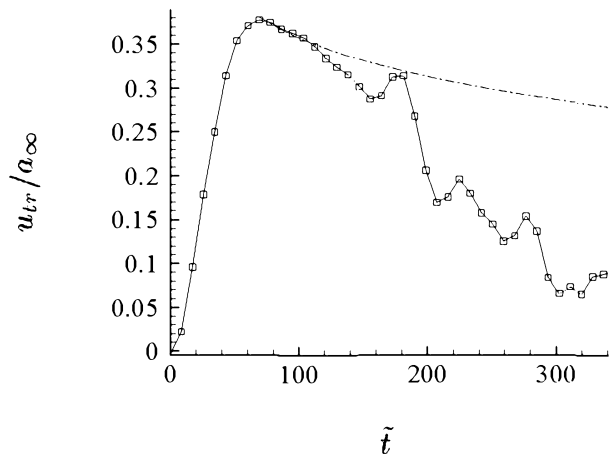

(b)

Figure 7. (a) One-dimensional energy spectra in $\theta$ direction. (-- - ) laminar stage, $(\square) \tilde{t}=\mathbf{8 . 6} ;(\Delta) \tilde{t}=43.2$; (o) $\tilde{t}=86.4 ;(\longrightarrow)$ turbulent stage, $(\square) \tilde{t}=130 ;(\triangle) \tilde{t}=216 ;$ (o) $\tilde{t}=302 ;$ (b) ( $\square$ ) ensemble averaged $(N=$ 12) translational velocity of the turbulent vortex ring with $M=0.28,(-\cdot-)$ of a laminar vortex ring with the same ring properties.

With least square fitting of Eq. (2), the virtual origins are found to be $\tilde{t}_{0}=130.0, x_{0}=0.064$. To test the self-similarity, we plot velocity profiles in both original $(x, r)$ and scaled coordinates $(\xi, \eta)$ in Figure 8 . The plots show that self-similar decay is achieved for $\tilde{t}>173$. Self-similarity of the sound field will be studied in the next section.

\section{The Acoustic Field}

The acoustic field is measured around a circle $|x|=16.1 R, \chi=52^{\circ}$ for a particular realization of the $M=0.28$ case, and is decomposed into azimuthal modes $n=0,1,2$. (Figure 9 (a)). A large pressure pulse is generated when the vortex ring is formed by the forcing. This acoustic transient is the same among all the realizations and can be removed by the ensemble average. The sound pressure level of the vortex ring peaks when the instability become nonlinear and the vortex ring starts to breakdown. The pressure signals have a distinct frequency at this stage. As the vortex ring transitions and vorticity begins to decay, both the amplitude and the frequency of the pressure disturbance decreases as well. The drift of the peak frequency to lower frequency is also detected in the experiments. ${ }^{12}$ It is also found that at this location, the amplitudes of the first 3 azimuthal modes are roughly equal. The contribution of higher modes to the total sound pressure level is negligible.

\section{A. Sound Pressure Level and Spectrum}

The sound pressure level (SPL) are measured and ensemble averaged at $\chi=52^{\circ}, 16.1 R_{0}$. This position corresponds to an acoustic delay of $\tilde{t}=58.0$ from the nominal ring position. The averaged SPL and SPL from 2 realizations are plotted against time in Figure 9 (b). For time comparison, stage I, II and III of the circulation evolution is marked. The sound generation also consists of three stages, which are related to the dynamics of the vortex ring. Before $\tilde{t}=200$, instability waves generate relatively weak sound, and the growth of the sound pressure level is related to the growth rate of the corresponding vortex ring instability 


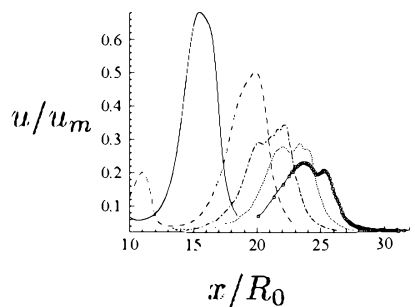

(a)

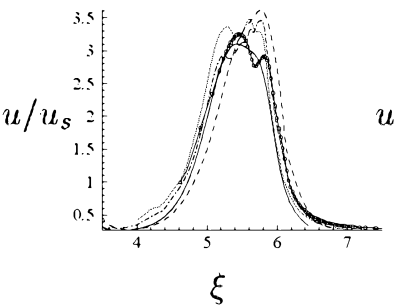

(b)

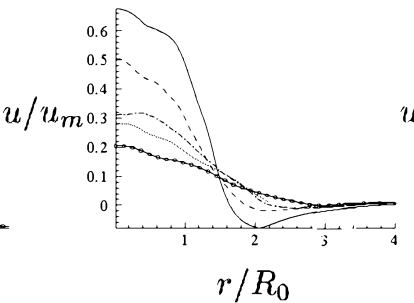

(c)

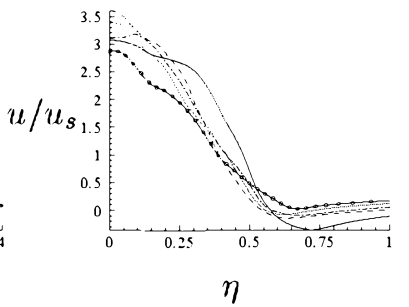

(d)

Figure 8. Centerline mean axial velocity. (a), in origin variables, scaled with the maximum axial velocity just after ring formation, $u_{m}$; (b) in similarity variables. Radial profile of mean axial velocity across the center of the vortex ring $(\mathbf{c})$ in origin variables, scaled with $u_{m} ;(\mathbf{d})$ in similarity variables. $(\longrightarrow) \tilde{t}=173(----) \tilde{t}=$ 216, (一- —) $\tilde{t}=\mathbf{2 5 9},(\cdots \cdots)) \tilde{t}=\mathbf{3 0 2},(\square) \tilde{t}=\mathbf{3 4 6}$.

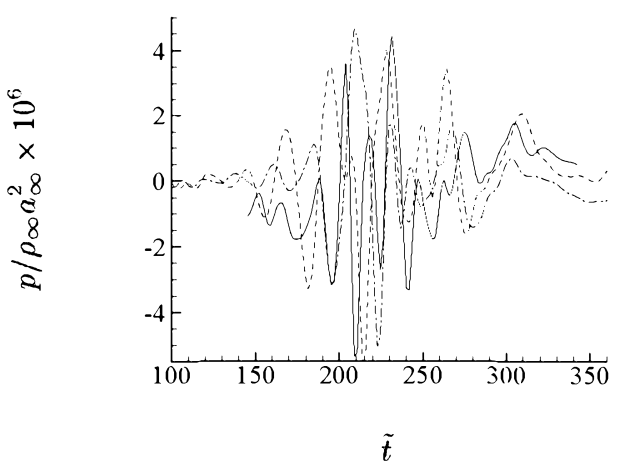

(a)

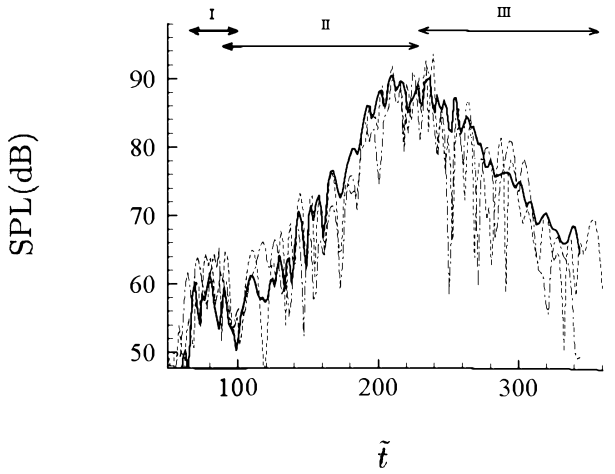

(b)

Figure 9. a) Azimuthal components of acoustic field at $|x|=16.1 R, \chi=52^{\circ},(\longrightarrow) n=\mathbf{0},(----) n=1$, $(--) n=2$, one realization in $M=0.28$ case; b) Sound pressure level measured at point $\chi=52^{\circ}, 16.1 R_{0}$, $(-)$ ensemble averaged $(N=12) ;(-\cdot-)$ realization $1 ;(----)$ realization 2. 
modes. From $\tilde{t}=200$ to 260 vortex breakdown occurs, and the sound pressure level is maximum. From $\tilde{t}=$ 260 to 300 , the turbulent decay of the ring leads to a decay in SPL by $30 \mathrm{~dB}$.

The spectrum at four different polar angles for azimuthal mode $n=0,1,2$ and the total is measured and plotted in Figure 10. Because the computational grids move with the vortex ring, there is no doppler shift in frequency at different angles. To compare with the experimental results of Zaitsev et. al. ${ }^{12}$ and the theoretical prediction of Kopiev et. al., ${ }^{18}$ the frequency is scaled with $f^{*}=\Omega_{0} / 4 \pi$, where $\Omega_{0}$ is the equivalent vorticity if vorticity is uniformly distributed inside the core. For a laminar vortex ring, the equivalent vorticity is calculated with the vortex ring translational velocity $U_{t r}$ and $\mu$, which is the ratio of core radius to ring radius, and the ring radius $R$, with the formula ${ }^{24}$

$$
\Omega_{0}=4 U_{t r} / \mu^{2} R C(\mu)
$$

where $C(\mu)=\log (8 / \mu)-0.25$.

The peak of the spectrum is found to be close to 1.0 , which agrees well with the value predicted by Kopiev's model, and the experimental results. The spectrum is also narrow banded, but the band width is larger than the experiments. A possible explanation can be obtained from the inviscid model. ${ }^{18}$ The bandwidth of the peak is proportional to $\mu$, and the vortex rings in the simulation have larger $\mu$ than the experiments. It is also demonstrated that at different polar angles, each mode has different relative strength. For example, at shallow angles to the vortex ring axis, the axisymmetric mode dominants; at higher angles, modes $n=1$ and 2 become relatively stronger, with mode $n=1$ becoming dominant at $\chi=55.9^{\circ}$. The measured directivity of the total sound field is the sum of contributions from each azimuthal modes, and is more monotonic than the individual components. Like the sound field of an unforced jet, there is no angle of extinction. This is a major difference between the sound field of a turbulent vortex ring and an axisymmetric vortex ring, or with axisymmetric interactions such as vortex ring pairing. ${ }^{25}$

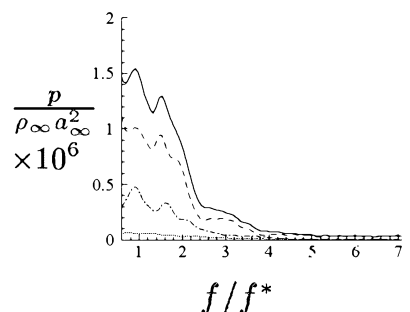

(a)

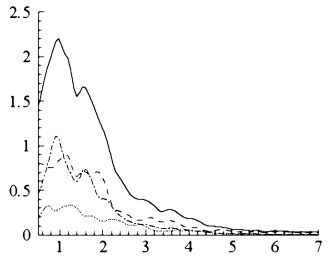

$f / f^{*}$

(b)

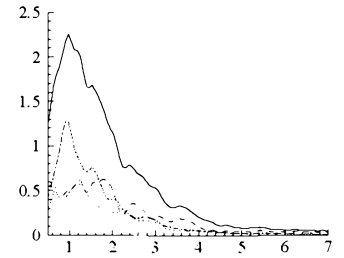

$f / f^{*}$

(c)

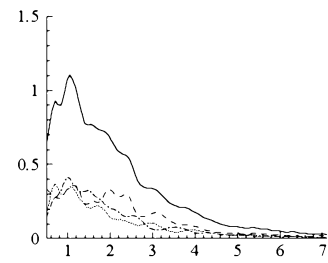

$f / f^{*}$

(d)

Figure 10. Spectrum of different azimuthal modes at different polar angles, $|x|=15 R$. a) $\chi=18.6^{\circ}$; b) $\chi=37.2^{\circ}$; c) $x=55.9^{\circ}$; d) $x=93.1^{\circ}$. ( $(\longrightarrow)$ total spectrum; $(---) n=\mathbf{0} ;(-\cdot-) n=\mathbf{1},(\cdots \cdots \cdots) n=2$.

\section{B. Comparison with Vortex Sound Theory}

From the vortex sound theory, ${ }^{14}$ the far-field sound generated by a compact vorticity field in a low-Mach number flow is

$$
p^{\prime}(\boldsymbol{x}, t)=\frac{\rho_{0}}{12 \pi c_{0}^{2}} \frac{x_{i} x_{j}}{|x|^{3}} \frac{d^{3}}{d t^{3}} Q_{i j}\left(t-|x| / c_{0}\right),
$$

where $Q_{i j}$ is given by the second-order moments of vorticity

$$
Q_{i j}(t)=\int(\boldsymbol{y} \times \boldsymbol{\omega}(\boldsymbol{y}, t))_{i} y_{j} d v
$$


To a first approximation, we examine the azimuthal component of vorticity. We can decompose the vorticity ficld into azimuthal Fourier modes

$$
\omega(r, \theta, z, t)=\sum_{k=-n}^{n} \omega_{k}(r, z, t) e^{i k \theta}
$$

It is found that all the moments with $n>2$ are zero. Pluging Eq. (6) into Eq. (4), we obtains, for $n=0,1,2$

$$
\begin{aligned}
& p_{0}(|x|, \chi)=\frac{\rho_{0}}{12 c_{0}^{2}} \frac{1}{|x|} \frac{d^{3} Q_{0}}{d t^{3}}\left(3 \cos ^{2} \chi-1\right), \\
& p_{1}(|x|, \chi, \theta)=\frac{\rho_{0}}{24 c_{0}^{2}} \frac{1}{|x|} \frac{d^{3} Q_{1}}{d t^{3}} \sin 2 \chi \cos \theta, \\
& p_{2}(|x|, \chi, \theta)=\frac{\rho_{0}}{24 c_{0}^{2}} \frac{1}{|x|} \frac{d^{3} Q_{2}}{d t^{3}} \sin ^{2} \chi \cos 2 \theta,
\end{aligned}
$$

where

$$
Q_{0}=\int r^{2} z \omega_{0} d z d r, \quad Q_{1}=\int\left(r z^{2}+r^{3}\right) \omega_{1} d z d r, \quad Q_{2}=\int r^{2} z \omega_{2} d z d r
$$

At finite translational Mach number, corrections to these formulas should be made, the pressure perturbation is intensified in the downstream direction. When we model the vortex ring as a simple quadrupole source convected with constant Mach number, $M$, the pressure perturbation will change by the factor $(1-M \cos \chi)^{-3}$.

Figure 11 (a) shows the polar distribution of sound pressure level associated with the total sound field, as well as contribution from azimuthal modes $n=0,1$, and 2. Also plotted as comparison is the convection amplification factor $p^{\prime} \sim(1-M \cos \chi)^{-3}$. When the convection term is suppressed from the sound pressure level, the directivity of each modes is closer to that predicted with Eq. (7), (8) and (9). The directivity for the first 3 azimuthal modes, and for two vortex ring Mach numbers $M=0.14$ and 0.28 , are depicted in Figure 11 (b) to (d), and are also compared with the theory. The pressure perturbation is scaled with $\rho_{0} M^{4} R /|x|(1-M \cos \chi)^{-3}$. The agreement between the simulation results and the simplified model is good. The deviation is likely due to the non-azimuthal components of the vorticity field, and the non-compactness of the acoustic source.

\section{Asymptotic Decay of the Sound Field}

Another interesting phenomena is the temporal decay of the sound pressure level after the transition. Since the flow becomes self-similar and the vorticity ficld evolves with a similarity law, the acoustic field generated by the vorticity field would also follow a similarity law.

From Eq. (4), at a fixed position in space $(|x|, \chi)$, the pressure perturbation changes with $\frac{d^{3}}{d t^{3}} Q_{i j}\left(t-x / c_{0}\right)$. In addition, as shown in Eq. (1), during the turbulent stage there is a velocity scale $u_{s}$ and a length scale $L_{s}$ upon which the flow is self-preserving.

Define nondimensional velocity $\boldsymbol{u}^{*}=\boldsymbol{u} / U_{s}, \boldsymbol{x}^{*}=\boldsymbol{x} / L_{s}$, which should be invariant in time, and then we get

$$
\boldsymbol{\omega}=\nabla \times \boldsymbol{u}=\frac{U_{s}}{L_{s}} \nabla^{*} \times \boldsymbol{u}^{*}=\left(t-t_{0}\right)^{-1} \boldsymbol{\omega}^{*}
$$

therefore

$$
\begin{aligned}
Q & =\int(\boldsymbol{x} \times \boldsymbol{\omega}(\boldsymbol{x}, t))_{i} x_{j} d v=L_{\boldsymbol{s}}^{5}\left(t-t_{0}\right)^{-1} \int\left(\boldsymbol{x}^{*} \times \boldsymbol{\omega}^{*}\left(\boldsymbol{x}^{*}, t\right)\right)_{i} x_{j}^{*} d v^{*} \\
& =\left(\frac{I}{\rho}\left(t-t_{0}\right)^{-\frac{1}{4}}\right) \int\left(\boldsymbol{x}^{*} \times \boldsymbol{\omega}^{*}\left(\boldsymbol{x}^{*}, t\right)\right)_{i} x_{j}^{*} d v^{*}
\end{aligned}
$$




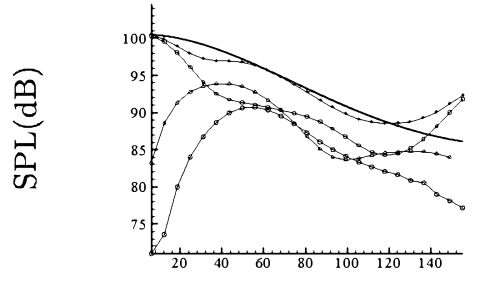

$\chi$

(a)

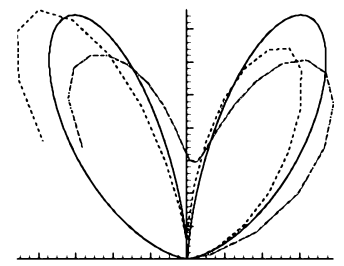

$p_{r m s}^{2}$

(c)

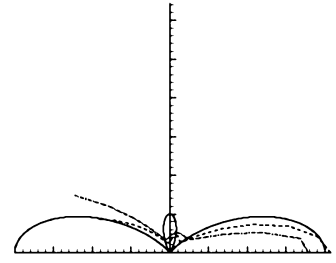

$p_{r m s}^{2}$

(b)

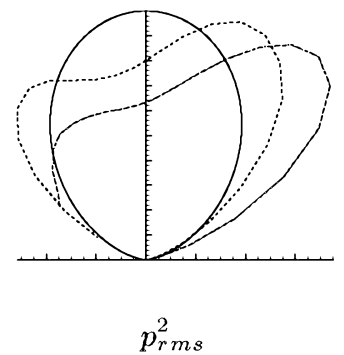

(d)

Figure 11. (a) Polar distribution of sound pressure level for $M=\mathbf{0 . 2 8}$ in $\mathbf{~ d B}$. $(\longrightarrow)(1-M \cos \chi)^{-3} \cdot(+)$ total pressure disturbance; $(\square) n=0 ;(\triangle) n=1 ;\left(\right.$ o) $n=2$. (b) to (d), Polar plot of $p_{r m s}^{2}$. (b) mode $n=0$, (一) $\left(3 \cos ^{2} \chi-1\right)^{2},(---) M=0.14,(--) M=0.28 ;$ (c) mode $n=1,(-) \sin ^{2} 2 \chi,(---) M=0.14$, (一. - $M=0.28$; (d) mode $n=2$, ( -$) \sin ^{4} \chi,(----) M=0.14,(-\cdot-) M=0.28$. Note that the theoretical directivities are plotted with an arbitrary overall amplitude. 
Finally

$$
\frac{d^{3} Q}{d t^{3}}=\left(t-t_{0}\right)^{-\frac{11}{4}} \int\left(\boldsymbol{x}^{*} \times \boldsymbol{\omega}^{*}\left(\boldsymbol{x}^{*}, t\right)\right)_{i} x_{j}^{*} d v^{*}
$$

and

$$
p^{2} \sim\left(\tilde{t}-\tilde{t}_{0}\right)^{-\frac{11}{2}}
$$

Figure 12 presents the decay of $p^{\prime 2}$ at point $\left(|x|=15 R_{0}, \chi=37.2^{\circ}\right)$ for two Mach numbers $M=0.14$ and 0.28 . The agreement with the similarity scales is satisfactory.

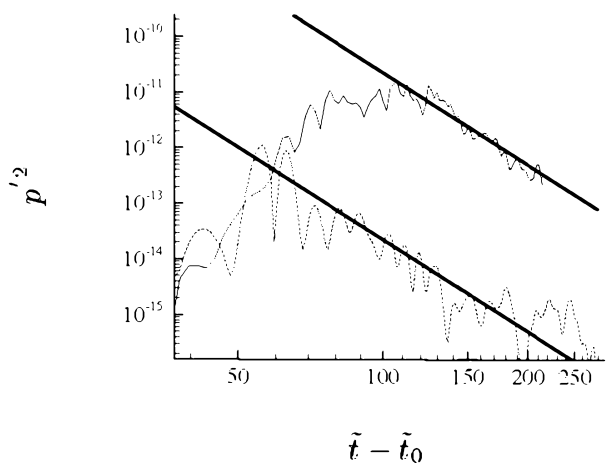

Figure 12. Decay of acoustic field at $\left(|x|=15 R_{0}, \chi=37.2^{\circ}\right)(-) \quad M=\mathbf{0 . 2 8}$, ensemble averaged $(N=12)$ $(---)) M=0.14$, ensemble averaged $(N=5) ;(\longrightarrow) p^{\prime 2} \sim\left(t-\tilde{t}_{0}\right)^{-\frac{11}{2}}$.

\section{Comparison with Jet Noise}

The spectrum, sound pressure level and directivity of the sound field from a turbulent vortex ring resemble the sound field of a turbulent jet. In this section we try to compute the sound field generated by a train of decorrelated vortex rings, as a notional model of sound generation from coherent structures in a turbulent jet. For a single vortex ring, sound radiation is the strongest when the vortex ring breaks down and transitions. The vortex breakdown takes place at an approximately fixed downstream location of about 7 to 8 ring diameters, which is approximately the same location of the close of the potential core in a low Reynolds number jet. When a train of de-correlated vortex rings breakdown consecutively at the same location, the sound detected in the far field is the sum of each vortex ring sound ficld, with a delay time $\Delta t$ between neighboring rings. We define a vortex ring replication Strouhal number $S t_{r}=2 R / u_{j} \Delta t$, where $u_{j}$ is the approximate exit jet velocity. A simple slug model predicts the translational velocity to be $U_{t r}=U_{p} / 2$, where $U_{p}$ is the piston velocity in the piston-cylinder mechanism, which is also the exit jet velocity from the cylinder. In practice, $U_{t r}$ varies from $0.5 U_{p}$ to $0.6 U_{p}$, therefore the vortex rings with $M=0.28$ correspond exit jet Mach number between 0.47 to 0.56 . Analogously, this is the approximate convection velocity of a large scale structure in a jet with $U_{t r}=0.5 U_{j}$ to $0.6 U_{j}$.

The pressure perturbations of 12 realizations are summed in random order with $S t_{r}$ varies from 0.08 to 0.4. The total spectrum is plotted in Figure 13 for two polar angles $30^{\circ}$ and $80^{\circ}$. At $S t_{r}=0.4$, the pressure perturbation has the most overlap, therefore higher amplitude; whereas at $S t_{r}<0.1$, there is no overlap of pressure perturbation and the spectrum is the same as an individual vortex ring. Between 0.2 and 0.33 , the spectrum is nearly invariant. On the other hand, the peak frequencies $S t_{j}=2 f R / u_{j}$ are between 0.4 to 0.5 , and are independent of the replication frequency. The spectrum at $\chi=30^{\circ}$ from experimental data ${ }^{26}$ of a Mach 0.9 jet with a Reynolds number $R e_{j}=U_{j} D_{j} / \nu=3600$ is overlaid on the spectrum in Figure 13 (a). 
The corresponding jet Reynolds number of the present vortex ring is 1900 . The peak frequency of the jet noise is about 0.23 , which is lower than the peak frequency of the vortex ring; however, the spectral shape at higher frequencies is quite similar between the jet and the train of vortex rings. In addition, the spectrum at $\chi=80^{\circ}$ is flatter than $\chi=30^{\circ}$, representing a larger contribution from higher frequency components.

In the simulations, the reference frame moves with the vortex ring at a constant velocity, but in most jet noise experiments, the acoustic field is measured at fixed locations. For better comparison with jet noise measurement, we interpolate the data from the grid that is moving with speed $M$ to a stationary grid. The sound pressure level at shallow angles will increase due to the translation of the vortex ring, whereas sound pressure level at downstream points will decrease.

To compare sound pressure level and directivity, the overall sound pressure level along a circle of $|x|=$ $15 R$ for the sound field of a single vortex ring in moving frame, in stationary frame, and a train of vortex rings in stationary frame are depicted in Figure 14. The sound pressure level at shallow angle in the stationary frame is higher than the moving frame, but decays faster downstream. When the sound fields of a train of vortex rings are replicated, the overall sound pressure level increases by about $5 \mathrm{~dB}$. We compare it to the sound field of a subsonic jet measured by Lush. ${ }^{27}$ The closest jet velocity in the experiments of Lush is 195 $\mathrm{m} / \mathrm{s}$, or Mach number 0.58 . The SPL of the jet noise is scaled from the distance of 120 nozzle diameters to 7.5 diameters. At $\chi=60^{\circ}$, the sound pressure level of the train of vortex rings is about $4 \mathrm{~dB}$ lower than the jet, likely due to slightly lower Mach number. To compare directivity, we plot the experimental data in Figure 14, with amplitude rescaled to agree with the train of vortex rings at $60^{\circ}$. The overall directivity patterns of the two curves are similar. A difference is that the sound radiation from the vortex rings is relatively stronger near the axis, and weaker at angle between $20^{\circ}$ to $40^{\circ}$, whereas in jet noise, the sound propagating at shallow angles is refracted to larger angles, thus increasing the sound pressure level in this region. The steeper fall off in directivity for $\chi>60^{\circ}$ in the simulation is probably a low Reynolds number effect and is similar to that observed in jet experiments ${ }^{26}$ and simulations. ${ }^{28}$ at low $R e$.

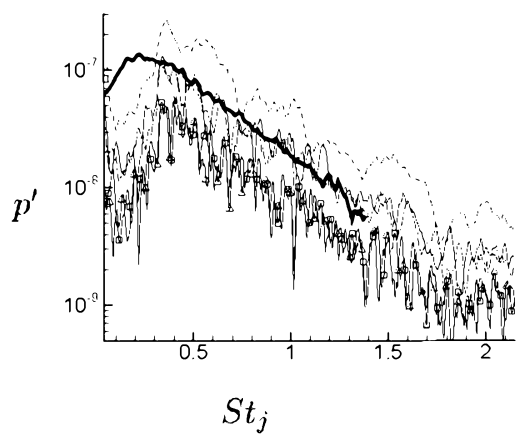

(a)

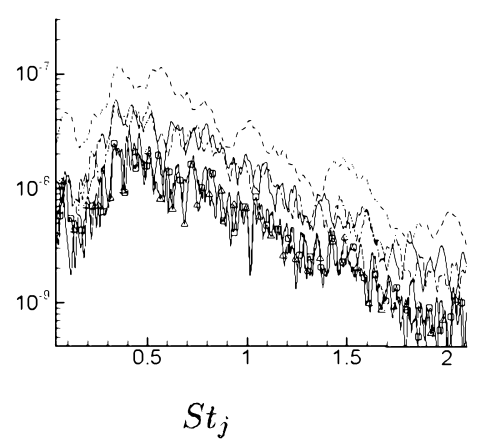

(b)

Figure 13. Spectrum at $x=60 R,(a) x=30^{\circ}$, (b) $\chi=80^{\circ}(----) S t_{r}=0.4 ;(\longrightarrow) S t_{r}=0.2 ;(-\cdot-) S t_{r}=$ $0.13 ;(\square) S t_{r}=0.1 ;(\Delta) S t_{r}=0.08,(\longrightarrow)$ from the experiment. ${ }^{26}$

\section{Conclusion}

We have computed transition and turbulent decay of an initially laminar vortex ring together with its radiated acoustic field. After an initial transient, instabilities with specific azimuthal mode numbers (primarily $n=6$ and $n=7$ ) and a particular radial structure grow most rapidly, in accord with linear stability theory. ${ }^{5}$ During later stages, nonlinear interactions bring about significant growth of the lower 


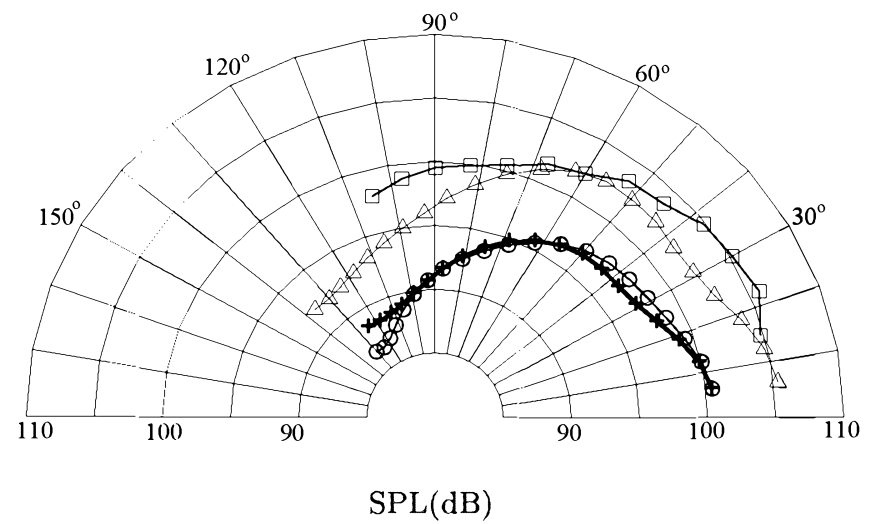

Figure 14. Polar distribution of total sound pressure level. $(+)$ turbulent vortex ring with translational Mach number $M=0.28$ along a circle of $|x|=15 R$ moving with the vortex ring, $(\circ)$ along a stationary circle; $(\triangle)$, a train of de-correlated vortex rings with $S t_{r}=0.2 ;(0)$ experimental data ${ }^{27}$ for a subsonic jet with jet Mach number 0.58 scaled to agree with the train of vortex rings at $60^{\circ}$.

azimuthal modes ( $n=0,1$, and 2 ) and lead to the strongest acoustic radiation. Eventually the vortex breaks down and the turbulence decays. During decay, we find a good collapse of the azimuthally and ensemble-averaged velocity fields with the self-similar theory. ${ }^{9}$

Statistics and spectra of the sound field are gathered for an ensemble of 12 realizations. The sound field is dominated by azimuthal modes $n=0,1$ and 2 (which are the only radiating modes if the noise source is compact). The directivity of these modes agrees well with predictions of vortex sound theory provided that we account for convective amplification due to ring translation. The spectra are peaked at a frequency that corresponds to $4 \pi f / \Omega_{0}=1.0$, in agreement with experiments. ${ }^{12}$ The theory of Kopiev et al. ${ }^{18}$ connects this frequency with linear eigen-oscillations of a thin-cored vortex ring.

Based on the theory for self-similar decay of the near-field, we use vortex sound theory to determine the decay rate for the acoustic field. Simulation results at two different Mach numbers confirm the scaling.

Finally, we compare sound radiated by the vortex ring to jet noise. We heuristically model the jet as a de-correlated train of vortex rings with a fixed replication frequency. To compare frequencies between the ring and jet, we assume that the ring translates with a convection speed corresponding to $0.5 U_{j}$ to $0.6 U_{j}$. We find that the directivity and shape of the noise spectrum is nearly invariant to replication frequencies around the range $S t=0.2$ to $S t=0.3$, which corresponds to the frequency of peak amplification of linear instability waves to the jet mean flow field. The primary effect of replication is to enhance SPL by about 5 $\mathrm{dB}$.

The peak frequency of the single vortex ring is preserved in the train of rings. It corresponds to about $S t=0.4$, which is somewhat higher than the peak frequency of jet noise, especially at low Mach and Reynolds numbers. Nevertheless, the spectral shape at higher frequencies is quite similar to low Reynolds number experiments ${ }^{26}$ for a $\mathrm{M}=0.9$ jet. The directivity also shows substantial agreement with jet noise data. The sound pressure level (of the replicated ring) is within about $4 \mathrm{~dB}$ of a higher-Reynolds number jet ${ }^{27}$ with a slightly higher Mach number than our simulations.

In future work we plan to further explore the connections between vortex ring and jet noise. The train of de-correlated rings appears to capture the main features of the low-Reynolds number acoustic ficld except perhaps the sound radiated by instabilities of the mean jet profile that lead to vortex roll-up upstream of the close of the potential core and the peak frequency with $S t=0.2$ to 0.3 . However, once formed, the vortex ring instabilities and breakdown may be reasonable models of the sound generation toward the close 
of the potential core. An intriguing possibility is that excitation of higher azimuthal modes in a jet (say with chevron nozzles) amplifies the most unstable modes (say with $n=6-7$ ) of vortices that are formed by the instabilities of the mean jet profile. This may lead to more rapid breakdown of the vorticity toward the close of the potential core in a process similar to that observer for the present isolated vortex ring.

\section{Acknowledgments}

This rescarch was supported in part by the National Science Foundation (Grant CTS-9501349).

\section{References}

1'Tam, C. and Auriault, L., "Jet mixing noise from fine-scale turbulence," AlA A Journal, Vol. 37, No. 2, 1999, pp. 145-153.

${ }^{2}$ Glezer, A., "The formation of vortex rings," Phys. Fluids, Vol. 31, 1988, pp. 3532 3541.

${ }^{3}$ Widnall, S. and Sullivan, J., "On the stability of vortex rings," Proc. R. Soc. London Ser. A, Vol. 332, 1973, pp. 335-353.

${ }^{4}$ Maxworthy, 'T., "Sorne experimental studies of vortex rings," J. Fluid Mech., Vol. 81, 1977, pp. 465 495.

"Widnall, S. and 'Tsai, C., "The instability of the thin vortex rings," Phil. Trans. R. Soc. London A, Vol. 287, 1977, pp. $273-305$

${ }^{6}$ Saffrnan, P., "The number of waves on unstable vortex rings," J. Fluid Mech., Vol. 84, 1978, pp. 625-639.

${ }^{7}$ Shariff, K., Verzicco, R., and Orlandi, P., "A numerical study of the three-dimensional vortex ring instabilities: viscous corrections and early nonlinear stage," J. Fluid Mech., Vol. 279. 1994, pp. 351 375.

${ }^{8}$ Knio, O. and Ghoniem, A., "Numerical Study of a Three-Dimensional Vortex Method," J. Comp. Physics, Vol. 86, 1990, pp. $75-106$.

${ }^{9}$ Glezer, A. and Coles, D., "An experimental study of a turbulent wortex ring," J. Fluid Mech., Vol. 211, 1990, pp. $243-283$.

${ }^{10}$ Weigand, A. and Gharib, M., "On the decay of a turbulent vortex ring," Phys. Fluids, Vol. 6, No. 12, 1994, pp. 3806 3808.

${ }^{11}$ Auerbach, D., "Stirring properties of vortex rings," Phys. Fluids, Vol. 3. No. 5, 1990, pp. 1351-1355.

${ }^{12}$ Zaitsev, M., Kopiev, V., Munin, A., and Potokin, A., "Sound radiation by a turbulent vortex ring," Sov. Phys. Dolk., Vol. 35, 1990, pp. 488-489.

${ }^{13}$ Zaitsev, M., Kopiev, V., and Kotova, A., "Representation of the Sound Field of a Turbulernt Vortex Ring as a Superposition of Quadrupoles," Acoustical Physics, Vol. 47, No. 6, 2001, pp. 793 801.

${ }^{14}$ Mohring, W., "On vortex sound at low Mach number," J. Fluid Mech., Vol. 85, 1978, pp. 685 691.

${ }^{15}$ Shariff, K., Leonard, A., and Ferziger, J., "Dynamics of a class of vortex rings," NASA TM 102257, NASA, 1989.

${ }^{16}$ Tang, S. and Ko, N., "Sound sources in the interactions of two inviscid two-dimensional vortex pairs." J. Fluid Mech., Vol. 419, 2000, pp. 117-201.

${ }^{1} \bar{\tau}^{\prime}$ ' ang, S. and Ko, N., "Basic sound generation mechanisms in inviscid vortex interactions at low Mach number," J. Sound Vib., Vol. 262, No. 1, 2003, pp. 87-115.

${ }^{18}$ Kopiev, V. and Chernyshev, S., "Vortex ring eigen-oscillations as a source of sound," J. Fluid Mech., Vol. $341,1997$. pp. 1957.

${ }^{19}$ Lele, S. K., "Compact finite difference schemes with spectral-like resolution," J. Comp. Physics, Vol. 103, No. 1, 1992, pp. 1642.

${ }^{20}$ Freund, J., "Proposed Inflow/Outfow Boundary Condition for Direct Computation of Aerodyramic Sound," AlAA J., Vol. 35, No. 4, 1997, pp. 740742.

${ }^{21}$ Mohseni, K. and Colonius, 'T., "Numerical treatment of polar coordinate singularities," J. Comp. Phys, Vol. 157, 2000, pp. $787-795$.

${ }^{22}$ Mohseni, K., Ran, H., and Colonius, 'T., "Numerical experiments on vortex ring formation." J. Fluid Mech., Vol. 430, 2001, pp. 267-282.

${ }^{23}$ Ran, H., "Numerical Study of the Dynamics and Sound Generation of a Turbulent Vortex Ring," Ph.d thesis, California Institute of 'Technology, 2004.

${ }^{24}$ Saffrnan, P., Vortex Dynamics, Cambridge University Press, 1992.

${ }^{25}$ Bridges, J. and Hussain, F., "Direct evaluation of aeroacoustic theory in a jet," J. Fluid Mech., Vol. 240, 1992, pp. 469-501.

${ }^{26}$ Stromberg, J., McLaughlin, D., and Troutt, 'T., "Flow field and acoustic properties of a Mach number 0.9 jet at a low Reynolds number," J. Sound Vib., Vol. 72, 1980, pr. 159-176.

${ }^{27}$ Lush, P., "Measurement of subsonic jet noise and comparison with theory." J. Fluid Mech.. Vol. 46, No. 3, 1971, pp. 477500 .

${ }^{28}$ Freund, J., "Noise sources in a low-Reynolds-number turbulent jet at Mach 0.9," J. Fluid Mech., Vol. 438, 2001, pp. 277305 .

16 of 16

American Institute of Aeronautics and Astronautics 